\title{
Strong convergence theorems for fixed point problems of infinite family of asymptotically quasi- $\phi$-nonexpansive mappings and a system of equilibrium problems
}

Xin Wang, Changsong Hu* and Jinlin Guan

\section{"Correspondence:}

huchang1004@yahoo.com.cn

School of Mathematics and

Statistics, Hubei Normal University,

Huangshi, 435002, China

\begin{abstract}
In this paper, we introduce a general iterative algorithm for finding a common element of the set of common fixed points of infinite family of asymptotically quasi- $\phi$-nonexpansive mappings and of the set of solutions for finite equilibrium problems in a real Banach space. Our results are the generalization of the results (Shehu in Comput. Math. Appl. 63:1089-1103, 2012; Kim in Fixed Point Theory Appl., 2011, doi:10.1186/1687-1812-2011-10) and (Kim and Buong in Fixed Point Theory Appl., 2011, doi:10.1155/2011/780764), and improvement of the result (Yang et al. in Appl. Math. Comput. 218:6072-6082, 2012).
\end{abstract}

MSC: $47 \mathrm{H} 09 ; 47 \mathrm{H} 10 ; 47 \mathrm{H} 17$

Keywords: common fixed point; asymptotically quasi- $\phi$-nonexpansive mappings; equilibrium problems; generalized $f$-projection operator

\section{Introduction}

Let $C$ be a nonempty, closed and convex subset of a real Banach space $E$. A mapping $T$ : $C \rightarrow C$ is called to be nonexpansive if

$$
\|T x-T y\| \leq\|x-y\|, \quad \forall x, y \in C .
$$

A mapping $T: C \rightarrow C$ is called to be quasi-nonexpansive if

$$
\left\|T x-x^{*}\right\| \leq\left\|x-x^{*}\right\|, \quad \forall x \in C, x^{*} \in F(T) .
$$

Let $F$ be a bifunction of $C \times C$ into $\mathbb{R}$. The equilibrium problem is to find $x \in C$ such that

$$
F(x, y) \geq 0, \quad \forall y \in C .
$$

The set of solutions to equilibrium problem (1.2) is denoted by $\operatorname{EP}(F)$. That is,

$$
\mathrm{EP}(F):=\{x \in C: F(x, y) \geq 0, \forall y \in C\} .
$$

\section{Springer}

(0) 2013 Wang et al.; licensee Springer. This is an Open Access article distributed under the terms of the Creative Commons Attribution License (http://creativecommons.org/licenses/by/2.0), which permits unrestricted use, distribution, and reproduction in any medium, provided the original work is properly cited. 
Recently, Yang et al. [1] proved strong convergence theorems for approximation of common fixed points of countably infinite family of asymptotically quasi- $\phi$-nonexpansive mappings in a uniformly smooth and strictly convex real Banach space, which has the Kadec-Klee property. More precisely, they proved the following theorem.

Theorem 1.1 Let E be a uniformly smooth and strictly convex Banach space, which has the Kadec-Klee property, and let $C$ be a nonempty closed convex subset of $E$. Let $G$ be a bifunction from $C \times C$ to $\mathbb{R}$ satisfying (A1)-(A4), and let $T_{i}: C \rightarrow C, \forall i \in \mathbb{N}$ be an infinite family of closed and asymptotically quasi- $\phi$-nonexpansive mapping with $\left\{k_{n i}\right\} \subset[1, \infty)$, $k_{n i} \rightarrow 1$ as $n \rightarrow \infty$, where $T_{0}=I$. Assume that $T_{i}, \forall i \in \mathbb{N}$ is asymptotically regular on $C$ and $\mathfrak{s}=\bigcap_{i=0}^{\infty} F\left(T_{i}\right) \cap \mathrm{EP}(G)$ is nonempty and bounded. Let $\left\{x_{n}\right\}$ be a sequence, generated by

$$
\left\{\begin{array}{l}
x_{0} \in E \quad \text { chosen arbitrarily, } \\
C_{1}=C, \\
x_{1}=\Pi_{C_{1} x_{0}}, \\
y_{n}=J^{-1}\left\{\sum_{i=0}^{\infty} \alpha_{n i} J T_{i}^{n} x_{n}\right\}, \\
u_{n} \in C \text { such that } G\left(u_{n}, y\right)+\frac{1}{r_{n}}\left\langle y-u_{n}, J u_{n}-J y_{n}\right\rangle \geq 0, \quad \forall y \in C, \\
C_{n+1}=\left\{z \in C_{n}: \phi\left(z, u_{n}\right) \leq \phi\left(z, x_{n}\right)+\left(k_{n}-1\right) M_{n}\right\}, \\
x_{n+1}=\Pi_{C_{n+1}} x_{0},
\end{array}\right.
$$

where $J$ is the duality mapping on $E, M_{n}=\sup \left\{\phi\left(z, x_{n}\right): z \in \Im\right\}$ for each $n \geq 1, k_{n}=$ $\sup _{i \geq 0}\left\{k_{n i}\right\},\left\{r_{n}\right\}$ is real sequence in $[a, \infty)$, where a is some positive real number, $\left\{\alpha_{n i}\right\}$ is a real sequence in $[0,1]$ satisfying the following conditions: (a) $\sum_{i=0}^{\infty} \alpha_{n i}=1, \forall n \geq 1$, (b) $\liminf _{n \rightarrow \infty} \alpha_{n 0} \alpha_{n i}>0, \forall i \in \mathbb{N}$. Then the sequence $\left\{x_{n}\right\}$ converges strongly to $\Pi_{\Im} x_{0}$.

In [2], Shehu introduced the following hybrid iterative scheme for approximating a common element of the set of fixed points of relatively quasi-nonexpansive mappings and the set of solutions to an equilibrium problem in a uniformly smooth and uniformly convex real Banach space: $x_{0} \in C, C_{1}=C, x_{1}=\Pi_{C_{1}}^{f} x_{0}$,

$$
\left\{\begin{array}{l}
y_{n}=J^{-1}\left(\alpha_{n} J x_{n}+\left(1-\alpha_{n}\right) J T_{n} x_{n}\right), \quad n \geq 1, \\
u_{n}=T_{r_{m, n}}^{F_{m}} T_{r_{m-1, n}}^{F_{m-1}} \cdots T_{r_{2, n}}^{F_{2}} T_{r_{1, n}}^{F_{1}} y_{n}, \\
C_{n+1}=\left\{w \in C_{n}: G\left(w, J u_{n}\right) \leq G\left(w, J x_{n}\right)\right\}, \quad n \geq 1, \\
x_{n+1}=\Pi_{C_{n+1}}^{f} x_{0}, \quad n \geq 1 .
\end{array}\right.
$$

Motivated by the facts above, the purpose of this paper is to prove a strong convergence theorem for finding a common element of the set of fixed points of asymptotically quasi$\phi$-nonexpansive mappings and the set of solutions to a system of equilibrium problems in a uniformly smooth and uniformly convex real Banach space, which has the Kadec-Klee property. 


\section{Preliminaries}

Let $E$ be a real Banach space, and let $E^{*}$ be the dual space of $E$. The duality mapping $J: E \rightarrow 2^{E^{*}}$ is defined by

$$
J(x)=\left\{f \in E^{*}:\langle x, f\rangle=\|x\|^{2}=\|f\|^{2}\right\} .
$$

By Hahn-Banach theorem, $J(x)$ is nonempty.

The modulus of smoothness of $E$ is the function $\rho_{E}:[0, \infty) \rightarrow[0, \infty)$ defined by

$$
\rho_{E}(\tau):=\sup \left\{\frac{1}{2}(\|x+y\|+\|x-y\|)-1:\|x\| \leq 1,\|y\| \leq \tau\right\} .
$$

$E$ is said to be uniformly smooth if $\lim _{\tau \rightarrow 0} \frac{\rho_{E}(\tau)}{\tau}=0$.

Let $\operatorname{dim} E \geq 2$. The modulus of convexity of $E$ is the function $\delta_{E}:(0,2] \rightarrow[0,1]$ defined by

$$
\delta_{E}(\epsilon):=\inf \left\{1-\left\|\frac{x-y}{2}\right\|:\|x\|=\|y\|=1 ; \epsilon=\|x-y\|\right\} .
$$

$E$ is said to be uniformly convex if $\forall \epsilon \in(0,2]$, there exists a $\delta=\delta(\epsilon)>0$ such that for $x, y \in E$ with $\|x\| \leq 1,\|y\| \leq 1$ and $\|x-y\| \geq \epsilon$, then $\left\|\frac{x+y}{2}\right\| \leq 1-\delta$. Equivalently, $E$ is uniformly convex if and only if $\delta_{E}(\epsilon)>0, \forall \epsilon \in(0,2]$. $E$ is strictly convex if for all $x, y \in E, x \neq y,\|x\|=$ $\|y\|=1$, we have $\|\lambda x+(1-\lambda) y\|<1, \forall \lambda \in(0,1)$.

It is well known that if $E$ is uniformly smooth, then $J$ is norm-to-norm uniformly continuous on each bounded subset of $E$. If $E$ is smooth, then $J$ is single-valued.

Recall that a Banach space $E$ has the Kadec-Klee property if for any sequence $\left\{x_{n}\right\} \subset E$ and $x \in E$ with $x_{n} \rightarrow x$ and $\left\|x_{n}\right\| \rightarrow\|x\|$, then $\left\|x_{n}-x\right\| \rightarrow 0$, as $n \rightarrow \infty$. It is well known that if $E$ is a uniformly convex Banach space, then $E$ has the Kadec-Klee property.

We denoted by $\phi$ the Lyapunov function from $E \times E$ to $\mathbb{R}$ defined by

$$
\phi(x, y):=\|x\|^{2}-2\langle x, J y\rangle+\|y\|^{2}, \quad \forall x, y \in E .
$$

It follows from the definition of $\phi$ that

$$
(\|x\|-\|y\|)^{2} \leq \phi(x, y) \leq(\|x\|+\|y\|)^{2}, \quad \forall x, y \in E .
$$

Let $E$ be a reflexive strictly convex and smooth Banach space. Then for $x, y \in E, \phi(x, y)=0$ if and only if $x=y$ (see $[3,4]$ ).

Definition 2.1 Let $C$ be a nonempty closed convex subset of $E$, and let $T$ be a mapping from $C$ into itself. A point $p \in C$ is said to be an asymptotic fixed point of $T$ if $C$ contains a sequence $\left\{x_{n}\right\}$, which converges weakly to $p$ and $\lim _{n \rightarrow \infty}\left\|x_{n}-T x_{n}\right\|=0$. The set of asymptotic fixed points of $T$ is denoted by $\widetilde{F}(T)$.

We say that $T$ is a relatively nonexpansive mapping [5-8] if the following conditions are satisfied:

(R1) $F(T) \neq \emptyset$;

(R2) $\phi(p, T x) \leq \phi(p, x), \forall x \in C, p \in F(T)$; 
(R3) $F(T)=\widetilde{F}(T)$.

If $T$ satisfies (R1) and (R2), then $T$ is said to be relatively quasi-nonexpansive [9-11].

Definition 2.2 We say that $T$ is an asymptotically $\phi$-nonexpansive mapping if there exists a sequence $\left\{k_{n}\right\} \subset[1, \infty)$ with $k_{n} \rightarrow 1$ as $n \rightarrow \infty$ such that $\phi\left(T^{n} x, T^{n} y\right) \leq k_{n} \phi(x, y)$, $\forall x, y \in C$. We say that $T$ is an asymptotically quasi- $\phi$-nonexpansive [11,12] mapping if $F(T) \neq \emptyset$ and there exists a sequence $\left\{k_{n}\right\} \subset[1, \infty)$ as $n \rightarrow \infty$ such that $\phi\left(p, T^{n} x\right) \leq$ $k_{n} \phi(p, x), \forall x \in C, p \in F(T)$.

It is easy to see that the class of relatively quasi-nonexpansive mappings and asymptotically quasi- $\phi$-nonexpansive mappings contains the class of relatively nonexpansive mappings. The class of asymptotically quasi- $\phi$-nonexpansive mappings is more general than the class of relatively quasi-nonexpansive mappings.

Following Alber [13], the generalized projection $\Pi_{C}: E \rightarrow C$ is defined by

$$
\Pi_{C}(x)=\left\{u \in C: \phi(u, x)=\min _{y \in C} \phi(y, x)\right\}, \quad \forall x \in E .
$$

The existence and uniqueness of the operator $\Pi_{C}$ follows from the properties of the function $\phi(y, x)$ and strict monotonicity of mapping $J$ (see, for example, $[3,4,13,14]$ ). If $E$ is a Hilbert space, then $\phi(y, x)=\|y-x\|^{2}, x, y \in E$ and $\Pi_{C}$ is the metric projection $P_{C}$ of $E$ onto $C$.

Next, we recall the concept and properties of generalized $f$-projector operator. Let $G$ : $C \times E^{*} \rightarrow \mathbb{R} \cup\{+\infty\}$ be a function defined as follows:

$$
G(\xi, \varphi)=\|\xi\|^{2}-2\langle\xi, \varphi\rangle+\|\varphi\|^{2}+2 \rho f(\xi)
$$

where $\xi \in C, \varphi \in E^{*}, \rho$ is a positive number, and $f: C \rightarrow \mathbb{R} \cup\{+\infty\}$ is proper, convex and lower semi-continuous. From the definitions of $G$ and $f$, it is easy to see that the following properties hold:

(i) $G(\xi, \varphi)$ is convex and continuous with respect to $\varphi$ when $\xi$ is fixed;

(ii) $G(\xi, \varphi)$ is convex and lower semi-continuous with respect to $\xi$ when $\varphi$ is fixed.

Definition 2.3 [15] Let $E$ be a real Banach space with its dual $E^{*}$. Let $C$ be a nonempty closed convex subset of $E$. We say that $\Pi_{C}^{f}: E^{*} \rightarrow 2^{C}$ is a generalized $f$-projection operator if

$$
\Pi_{C}^{f} \varphi=\left\{u \in C: G(u, \varphi)=\inf _{\xi \in C} G(\xi, \varphi)\right\}, \quad \forall \varphi \in E^{*}
$$

Lemma 2.4 [15] Let E be a reflexive Banach space with its dual $E^{*}$. Let $C$ be a nonempty closed convex subset of $E$. Then the following statements hold:

(i) $\Pi_{C}^{f} \varphi$ is a nonempty closed convex subset of $C$ for all $\varphi \in E^{*}$;

(ii) If $E$ is smooth, then for all $\varphi \in E^{*}, x \in \Pi_{C}^{f} \varphi$ if and only if

$$
\langle x-y, \varphi-J x\rangle+\rho f(y)-\rho f(x) \geq 0, \quad \forall y \in C
$$

(iii) [16] If $E$ is strictly convex, then $\Pi_{C}^{f}$ is a single-valued mapping. 
Recall that $J$ is a single-valued mapping when $E$ is a smooth Banach space. There exists a unique element $\varphi \in E^{*}$ such that $\varphi=J x$ for each $x \in E$. This substitution in (2.3) gives

$$
G(\xi, J x)=\|\xi\|^{2}-2\langle\xi, J x\rangle+\|x\|^{2}+2 \rho f(\xi)
$$

Now, we consider the second generalized $f$-projection operator in Banach space.

Definition 2.5 Let $E$ be a real Banach space and $C$ be a nonempty closed convex subset of $E$. We say that $\Pi_{C}^{f}: E \rightarrow 2^{C}$ is a generalized $f$-projection operator if

$$
\Pi_{C}^{f} x=\left\{u \in C: G(u, J x)=\inf _{\xi \in C} G(\xi, J x)\right\}, \quad \forall x \in E .
$$

Obviously, the definition of relatively quasi-nonexpansive mapping $T$ is equivalent to

(R'1) $F(T) \neq \emptyset$;

$\left(\mathrm{R}^{\prime} 2\right) \quad G(p, J T x) \leq G(p, J x), \forall x \in C, p \in F(T)$.

Lemma 2.6 [17] Let $C$ be a nonempty, closed and convex subset of a smooth and reflexive Banach space E. Then the following statements hold:

(i) $\Pi_{C}^{f} x$ is a nonempty closed convex subset of $C$ for all $x \in E$;

(ii) For all $x \in E, \hat{x} \in \Pi_{C}^{f} x$ if and only if

$$
\langle\hat{x}-y, J x-J \hat{x}\rangle+\rho f(y)-\rho f(\hat{x}) \geq 0, \quad \forall y \in C ;
$$

(iii) [16] If E is strictly convex, then $\Pi_{C}^{f} x$ is a single-valued mapping.

Lemma 2.7 [18] Let $E$ be a Banach space, and $f: E \rightarrow \mathbb{R} \cup\{+\infty\}$ is convex and lower semi-continuous. Then there exists $x^{*} \in E^{*}$ and $\alpha \in \mathbb{R}$ such that

$$
f(x) \geq\left\langle x, x^{*}\right\rangle+\alpha, \quad \forall x \in E
$$

Lemma 2.8 [17] Let $C$ be a nonempty closed convex subset of a smooth and reflexive Banach space $E$. Let $x \in E$ and $\hat{x} \in \Pi_{C}^{f} x$. Then

$$
\phi(y, \hat{x})+G(\hat{x}, J x) \leq G(y, J x), \quad \forall y \in C
$$

Lemma 2.9 [1, 19] Let E be a uniformly smooth and strictly convex Banach space, which has the Kadec-Klee property, and let $C$ be a nonempty closed convex subset of E. Let $T$ be a closed and asymptotically quasi- $\phi$-nonexpansive mapping. Then $F(T)$ is a closed and convex subset of $C$.

Lemma 2.10 [1] Let E be a uniformly convex real Banach space. For arbitrary $r>0$, let $B_{r}(0):=\{x \in E:\|x\| \leq r\}$. Then, for any given sequence $\left\{x_{n}\right\}_{n=1}^{\infty} \subset B_{r}(0)$ and for any given sequence $\left\{\lambda_{n}\right\}_{n=1}^{\infty}$ of positive numbers such that $\sum_{i=1}^{\infty} \lambda_{i}=1$, there exists a continuous strictly increasing convex function $g:[0,2 r] \rightarrow \mathbb{R}, g(0)=0$ such that for any positive integers $i, j$ with $i<j$, the following inequality holds

$$
\left\|\sum_{n=1}^{\infty} \lambda_{n} x_{n}\right\|^{2} \leq \sum_{n=1}^{\infty} \lambda_{n}\left\|x_{n}\right\|^{2}-\lambda_{i} \lambda_{j} g\left(\left\|x_{i}-x_{j}\right\|\right) .
$$


Lemma 2.11 [17] Let $E$ be a Banach space and $y \in E$. Let $f: E \rightarrow \mathbb{R} \cup\{+\infty\}$ be a proper, convex and lower semi-continuous mapping with convex domain $D(f)$.If $\left\{x_{n}\right\}$ is a sequence in $D(f)$ such that $x_{n} \rightarrow x \in \operatorname{int}(D(f))$ and $\lim _{n \rightarrow \infty} G\left(x_{n}, J y\right)=G(x, J y)$, then $\lim _{n \rightarrow \infty}\left\|x_{n}\right\|=$ $\|x\|$.

For solving the equilibrium problem for a bifunction $F: C \times C \rightarrow \mathbb{R}$, let us assume that $F$ satisfies the following conditions:

(A1) $F(x, x)=0$ for all $x \in C$;

(A2) $F$ is monotone, i.e., $F(x, y)+F(y, x) \leq 0$ for all $x, y \in C$;

(A3) for each $x, y, z \in C, \lim _{t \rightarrow 0} F(t z+(1-t) x, y) \leq F(x, y)$;

(A4) for each $x \in C, y \mapsto F(x, y)$ is convex and lower semicontinuous.

Lemma 2.12 [20] Let $C$ be a nonempty closed convex subset of a smooth, strictly convex and reflexive Banach space $E$, and let $F$ be a bifunction of $C \times C$ into $\mathbb{R}$ satisfying (A1)-(A4). Let $r>0$ and $x \in E$. Then, there exists $z \in C$ such that

$$
F(z, y)+\frac{1}{r}\langle y-z, J z-J x\rangle \geq 0, \quad \forall y \in C .
$$

Lemma 2.13 [9, 21] Let C be a nonempty closed convex subset of a smooth, strictly convex and reflexive Banach space $E$, and let $F$ be a bifunction of $C \times C$ into $\mathbb{R}$ satisfying (A1)-(A4). Let $r>0$ and $x \in E$. Define a mapping $T_{r}^{F}: E \rightarrow C$ as follows:

$$
T_{r}^{F}(x)=\left\{z \in C: F(z, y)+\frac{1}{r}\langle y-z, J z-J x\rangle \geq 0, \forall y \in C\right\}
$$

for all $z \in E$. Then, the following hold:

1. $T_{r}^{F}$ is single-valued;

2. $T_{r}^{F}$ is firmly nonexpansive mapping, i.e., for any $x, y \in E$,

$$
\left\langle T_{r}^{F} x-T_{r}^{F} y, J T_{r}^{F} x-J T_{r}^{F} y\right\rangle \leq\left\langle T_{r}^{F} x-T_{r}^{F} y, J x-J y\right\rangle
$$

3. $F\left(T_{r}^{F}\right)=\mathrm{EP}(F)$;

4. $T_{r}^{F} x$ is relatively quasi-nonexpansive;

5. $\mathrm{EP}(F)$ is closed and convex.

Lemma 2.14 [21] Let C be a nonempty closed convex subset of a smooth, strictly convex and reflexive Banach space $E$, and let $F$ be a bifunction of $C \times C$ into $\mathbb{R}$ satisfying (A1)-(A4). Let $r>0$. Then for each $x \in E$ and $q \in F\left(T_{r}^{F}\right)$,

$$
\phi\left(q, T_{r}^{F} x\right)+\phi\left(T_{r}^{F} x, x\right) \leq \phi(q, x) .
$$

An operator $T$ in a Banach space $E$ is said to be closed if $x_{n} \rightarrow x$ and $T x_{n} \rightarrow y$, then $T x=y$.

\section{Main result}

Theorem 3.1 Let E be a uniformly smooth and strictly convex Banach space, which has the Kadec-Klee property, and let $C$ be a nonempty closed convex subset of $E$. For each $k=$ 
$1,2, \ldots, m$, let $F_{k}$ be a bifunction from $C \times C$ satisfying (A1)-(A4), and let $\left\{T_{i}\right\}_{i=0}^{\infty}: C \rightarrow C$, $\forall i \in \mathbb{N}$ be an infinite family of closed and asymptotically quasi- $\phi$-nonexpansive mappings with sequence $\left\{k_{n i}\right\} \subset[1, \infty), k_{n i} \rightarrow 1$ as $n \rightarrow \infty$, where $T_{0}=I$. Assume that $T_{i}, \forall i \in \mathbb{N}$ is asymptotically regular on $C$ and $\Omega=\left(\bigcap_{i=0}^{\infty} F\left(T_{i}\right)\right) \cap\left(\bigcap_{k=1}^{m} \operatorname{EP}\left(F_{k}\right)\right)$ is nonempty and bounded. Let $f: E \rightarrow \mathbb{R}$ be a convex and lower semicontinuous mapping with $C \subset \operatorname{int}(D(f))$, and suppose that $\left\{x_{n}\right\}_{n=0}^{\infty}$ is a sequence generated by $x_{0} \in C, C_{1}=C, x_{1}=\Pi_{C_{1}}^{f} x_{0}$,

$$
\left\{\begin{array}{l}
y_{n}=J^{-1}\left\{\sum_{i=0}^{\infty} \alpha_{n i} J T_{i}^{n} x_{n}\right\}, \\
u_{n}=T_{r_{m, n}}^{F_{m}} T_{r_{m-1, n}}^{F_{m-1}} \cdots T_{r_{2, n}}^{2_{2}} T_{r_{1, n}}^{F_{1}} y_{n}, \\
C_{n+1}=\left\{z \in C_{n}: G\left(z, J u_{n}\right) \leq G\left(z, J x_{n}\right)+\left(k_{n}-1\right) M_{n}\right\} \\
x_{n+1}=\Pi_{C_{n+1}}^{f} x_{0},
\end{array}\right.
$$

where $J$ is the duality mapping on $E, M_{n}=\sup \left\{\phi\left(z, x_{n}\right): z \in \Omega\right\}$ for each $n \geq 1, k_{n}=$ $\sup _{i \geq 0}\left\{k_{n i}\right\},\left\{\alpha_{n i}\right\}$ is a real sequence in $[0,1]$ and $\left\{r_{k, n}\right\}_{n=1}^{\infty} \subset(0, \infty), k=1,2, \ldots, m$, satisfying the following conditions:
(a) $\sum_{i=0}^{\infty} \alpha_{n i}=1, \quad \forall n \geq 1$;
(b) $\quad \liminf _{n \rightarrow \infty} \alpha_{n 0} \alpha_{n i}>0, \quad \forall i \in \mathbb{N}$;
(c) $\liminf _{n \rightarrow \infty} r_{k, n}>0$.

Then the sequence $\left\{x_{n}\right\}$ converges strongly to $\Pi_{\Omega}^{f} x_{0}$.

Proof Step 1. We first show that $C_{n}, \forall n \geq 1$ is nonempty, closed and convex.

Now, we show that $C_{n}, \forall n \geq 1$ is closed and convex. It is obvious that $C_{1}=C$ is closed and convex. Suppose that $C_{n}$ is closed convex for some $n>1$. From the definition of $C_{n+1}$, we have $z \in C_{n+1}$, which implies that $G\left(z, J u_{n}\right) \leq G\left(z, J x_{n}\right)+\left(k_{n}-1\right) M_{n}$. This is equivalent to

$$
2\left(\left\langle z, J x_{n}\right\rangle-\left\langle z, J u_{n}\right\rangle\right) \leq\left\|x_{n}\right\|^{2}-\left\|u_{n}\right\|^{2}+\left(k_{n}-1\right) M_{n} .
$$

This implies that $C_{n+1}$ is closed convex for the same $n>1$. Hence, $C_{n}$ is closed and convex $\forall n \geq 1$.

By taking $\theta_{n}^{k}=T_{r_{k, n}}^{F_{k}} T_{r_{k-1, n}}^{F_{k-1}} \ldots T_{r_{2, n}}^{F_{2}} T_{r_{1, n}}^{F_{1}}, k=1,2, \ldots, m$ and $\theta_{n}^{0}=I$ for all $n \geq 1$, we obtain $u_{n}=\theta_{n}^{m} y_{n}$.

We next show that $\Omega \subset C_{n}, \forall n \geq 1$. From Lemma 2.13, we have that $T_{r_{k, n}}^{F_{k}}, k=1,2, \ldots, m$ is relatively nonexpansive mapping. For $n=1$, we have $\Omega \subset C_{1}=C$. Now, assume that $\Omega \subset C_{n}$ for some $n>1$. For each $x^{*} \in \Omega$, we obtain

$$
\begin{aligned}
G\left(x^{*}, J u_{n}\right) & =G\left(x^{*}, J \theta_{n}^{m} y_{n}\right) \leq G\left(x^{*}, J y_{n}\right) \\
& =\left\|x^{*}\right\|^{2}-2\left\langle x^{*}, J y_{n}\right\rangle+\left\|y_{n}\right\|^{2}+2 \rho f\left(x^{*}\right) \\
& \leq\left\|x^{*}\right\|^{2}-2 \sum_{i=0}^{\infty} \alpha_{n i}\left|x^{*}, J T_{i}^{n} x_{n}\right\rangle+\sum_{i=0}^{\infty} \alpha_{n i}\left\|T_{i}^{n} x_{n}\right\|^{2}+2 \rho f\left(x^{*}\right)
\end{aligned}
$$




$$
\begin{aligned}
& =\sum_{i=0}^{\infty} \alpha_{n i} \phi\left(x^{*}, T_{i}^{n} x_{n}\right)+2 \rho f\left(x^{*}\right) \\
& \leq \sum_{i=0}^{\infty} \alpha_{n i} k_{n i} \phi\left(x^{*}, x_{n}\right)+2 \rho f\left(x^{*}\right) \\
& =\sum_{i=0}^{\infty} \alpha_{n i}\left(1+\left(k_{n i}-1\right)\right) \phi\left(x^{*}, x_{n}\right)+2 \rho f\left(x^{*}\right) \\
& =G\left(x^{*}, J x_{n}\right)+\sum_{i=0}^{\infty} \alpha_{n i}\left(k_{n i}-1\right) \phi\left(x^{*}, x_{n}\right) \\
& \leq G\left(x^{*}, J x_{n}\right)+\left(k_{n}-1\right) M_{n} .
\end{aligned}
$$

So, $x^{*} \in C_{n+1}$. It implies that $\Omega \subset C_{n}, \forall n \geq 1$, and the sequence $\left\{x_{n}\right\}_{n=0}^{\infty}$ generated by (3.1) is well defined.

Step 2. We show that $\lim _{n \rightarrow \infty} G\left(x_{n}, J x_{0}\right)$ exists.

Since $f: E \rightarrow \mathbb{R}$ is a convex and lower semi-continuous, applying Lemma 2.7, we see that there exist $u^{*} \in E^{*}$ and $\alpha \in \mathbb{R}$ such that

$$
f(y) \geq\left\langle y, u^{*}\right\rangle+\alpha, \quad \forall y \in E .
$$

It follows that

$$
\begin{aligned}
G\left(x_{n}, J x_{0}\right) & =\left\|x_{n}\right\|^{2}-2\left\langle x_{n}, J x_{0}\right\rangle+\left\|x_{0}\right\|^{2}+2 \rho f\left(x_{n}\right) \\
& \geq\left\|x_{n}\right\|^{2}-2\left\langle x_{n}, J x_{0}\right\rangle+\left\|x_{0}\right\|^{2}+2 \rho\left\langle x_{n}, u^{*}\right\rangle+2 \rho \alpha \\
& =\left\|x_{n}\right\|^{2}-2\left\langle x_{n}, J x_{0}-\rho u^{*}\right\rangle+\left\|x_{0}\right\|^{2}+2 \rho \alpha \\
& \geq\left\|x_{n}\right\|^{2}-2\left\|x_{n}\right\|\left\|J x_{0}-\rho u^{*}\right\|+\left\|x_{0}\right\|^{2}+2 \rho \alpha \\
& =\left(\left\|x_{n}\right\|-\left\|J x_{0}-\rho u^{*}\right\|\right)^{2}+\left\|x_{0}\right\|^{2}-\left\|J x_{0}-\rho u^{*}\right\|^{2}+2 \rho \alpha .
\end{aligned}
$$

Since $x_{n}=\Pi_{C_{n}}^{f} x_{0}$, it follows from (3.3) that

$$
G\left(x^{*}, J x_{0}\right) \geq G\left(x_{n}, J x_{0}\right) \geq\left(\left\|x_{n}\right\|-\left\|J x_{0}-\rho u^{*}\right\|\right)^{2}+\left\|x_{0}\right\|^{2}-\left\|J x_{0}-\rho u^{*}\right\|^{2}+2 \rho \alpha
$$

for each $x^{*} \in F(T)$. This implies that $\left\{x_{n}\right\}_{n=0}^{\infty}$ is bounded and so is $\left\{G\left(x_{n}, J x_{0}\right)\right\}_{n=0}^{\infty}$. By the construction of $C_{n}$, we have that $C_{n+1} \subset C_{n}$ and $x_{n+1}=\Pi_{C_{n+1}}^{f} x_{0} \in C_{n}$. It follows from Lemma 2.8 that

$$
\phi\left(x_{n+1}, x_{n}\right)+G\left(x_{n}, J x_{0}\right) \leq G\left(x_{n+1}, J x_{0}\right) .
$$

It is obvious that

$$
\phi\left(x_{n+1}, x_{n}\right) \geq\left(\left\|x_{n+1}\right\|-\left\|x_{n}\right\|\right)^{2} \geq 0,
$$

and so, $\left\{G\left(x_{n}, J x_{0}\right)\right\}_{n=0}^{\infty}$ is nondecreasing. It follows that the limit of $\left\{G\left(x_{n}, J x_{0}\right)\right\}_{n=0}^{\infty}$ exists.

Step 3. We prove that $\lim _{n \rightarrow \infty}\left\|J x_{n}-J T_{j}^{n} x_{n}\right\|=0, \forall j \in \mathbb{N}$. 
Now, since $\left\{x_{n}\right\}_{n=0}^{\infty}$ is bounded in $C$, and $E$ is reflexive, we may assume that $x_{n} \rightarrow p$, and since $C_{n}$ is closed and convex for each $n \geq 1$, it is easy to see that $p \in C_{n}$ for each $n \geq 1$. Again, since $x_{n}=\Pi_{C_{n}}^{f} x_{0}$, we obtain

$$
G\left(x_{n}, J x_{0}\right) \leq G\left(p, J x_{0}\right), \quad \forall n \geq 0 .
$$

Since

$$
\begin{aligned}
\liminf _{n \rightarrow \infty} G\left(x_{n}, J x_{0}\right) & =\liminf _{n \rightarrow \infty}\left\{\left\|x_{n}\right\|^{2}-2\left\langle x_{n}, J x_{0}\right\rangle+\left\|x_{0}\right\|^{2}+2 \rho f\left(x_{n}\right)\right\} \\
& \geq\|p\|^{2}-2\left\langle p, J x_{0}\right\rangle+\left\|x_{0}\right\|^{2}+2 \rho f(p)=G\left(p, J x_{0}\right) .
\end{aligned}
$$

Then, we obtain

$$
G\left(p, J x_{0}\right) \leq \liminf _{n \rightarrow \infty} G\left(x_{n}, J x_{0}\right) \leq \limsup _{n \rightarrow \infty} G\left(x_{n}, J x_{0}\right) \leq G\left(p, J x_{0}\right)
$$

This implies that

$$
\lim _{n \rightarrow \infty} G\left(x_{n}, J x_{0}\right)=G\left(p, J x_{0}\right)
$$

By Lemma 2.11, we obtain that $\lim _{n \rightarrow \infty}\left\|x_{n}\right\|=\|p\|$. In view of Kadec-Klee property of $E$, we have that $\lim _{n \rightarrow \infty} x_{n}=p$.

By the construction of $C_{n}$, we have that $C_{n+1} \subset C_{n}$ and $x_{n+1}=\Pi_{C_{n+1}}^{f} x_{0} \in C_{n+1}$. It follows that

$$
\phi\left(x_{n+1}, u_{n}\right) \leq \phi\left(x_{n+1}, x_{n}\right)+\left(k_{n}-1\right) M_{n} .
$$

Now, (3.4) implies that

$$
\phi\left(x_{n+1}, u_{n}\right) \leq \phi\left(x_{n+1}, x_{n}\right)+\left(k_{n}-1\right) M_{n} \leq G\left(x_{n+1}, J x_{0}\right)-G\left(x_{n}, J x_{0}\right)+\left(k_{n}-1\right) M_{n} .
$$

Taking the limit as $n \rightarrow \infty$ in (3.5), we obtain

$$
\lim _{n \rightarrow \infty} \phi\left(x_{n+1}, x_{n}\right)=0
$$

Therefore,

$$
\lim _{n \rightarrow \infty} \phi\left(x_{n+1}, u_{n}\right)=0
$$

It then yields that $\lim _{n \rightarrow \infty}\left(\left\|x_{n+1}\right\|-\left\|u_{n}\right\|\right)=0$. Since $\lim _{n \rightarrow \infty}\left\|x_{n+1}\right\|=\|p\|$, we have

$$
\lim _{n \rightarrow \infty}\left\|u_{n}\right\|=\|p\|
$$

Hence,

$$
\lim _{n \rightarrow \infty}\left\|J u_{n}\right\|=\|J p\|
$$

This implies that $\left\{\left\|J u_{n}\right\|\right\}_{n=0}^{\infty}$ is bounded in $E^{*}$. Since $E$ is reflexive, and so $E^{*}$ is reflexive, we can then assume that $J u_{n} \rightarrow f_{0} \in E^{*}$. In view of reflexivity of $E$, we see that $J(E)=E^{*}$. 
Hence, there exists $x \in E$ such that $J x=f_{0}$. Since

$$
\phi\left(x_{n+1}, u_{n}\right)=\left\|x_{n+1}\right\|^{2}-2\left\langle x_{n+1}, J u_{n}\right\rangle+\left\|u_{n}\right\|^{2}=\left\|x_{n+1}\right\|^{2}-2\left\langle x_{n+1}, J u_{n}\right\rangle+\left\|J u_{n}\right\|^{2} .
$$

Taking $\liminf \operatorname{in}_{n \rightarrow \infty}$ for both sides of the equality above, yields that

$$
0 \geq\|p\|^{2}-2\left\langle p, f_{0}\right\rangle+\left\|f_{0}\right\|^{2}=\|p\|^{2}-2\langle p, J x\rangle+\|J x\|^{2}=\|p\|^{2}-2\langle p, J x\rangle+\|x\|^{2}=\phi(p, x) .
$$

That is, $p=x$. This implies that $f_{0}=J p$, and so, $J u_{n} \rightarrow J p$. It follows from $\lim _{n \rightarrow \infty}\left\|J u_{n}\right\|=$ $\|J p\|$ and Kadec-Klee property of $E^{*}$ (this is because $E^{*}$ is uniformly convex) that

$$
J u_{n} \rightarrow J p
$$

Note that $J^{-1}: E^{*} \rightarrow E$ is hemi-continuous (this is because $E$ is a uniformly smooth and strictly convex Banach space with a strictly convex dual $\left.E^{*}\right)$, it follows that $u_{n} \rightarrow p$. Since (3.6) and $E$ have the Kadec-Klee property, we obtain that

$$
\lim _{n \rightarrow \infty} u_{n}=p
$$

It follows that

$$
\lim _{n \rightarrow \infty}\left\|x_{n}-u_{n}\right\|=0
$$

Since $J$ is uniformly norm-to-norm continuous on any bounded sets, we have

$$
\lim _{n \rightarrow \infty}\left\|J x_{n}-J u_{n}\right\|=0
$$

Let $r:=\sup _{n, i \geq 0}\left\{\left\|T_{i}^{n} x_{n}\right\|\right\}$. Since $E$ is uniformly smooth, we know that $E^{*}$ is uniformly convex. Then from Lemma 2.10 , we have

$$
\begin{aligned}
G\left(x^{*}, J u_{n}\right)= & G\left(x^{*}, J \theta_{n}^{m} y_{n}\right) \\
\leq & G\left(x^{*}, J y_{n}\right) \\
= & \left\|x^{*}\right\|^{2}-2\left\langle x^{*}, \sum_{i=0}^{\infty} \alpha_{n i} J T_{i}^{n} x_{n}\right\rangle+\left\|\sum_{i=0}^{\infty} \alpha_{n i} J T_{i}^{n} x_{n}\right\|^{2}+2 \rho f\left(x^{*}\right) \\
\leq & \left\|x^{*}\right\|^{2}-2 \sum_{i=0}^{\infty} \alpha_{n i}\left\langle x^{*}, J T_{i}^{n} x_{n}\right\rangle+\sum_{i=0}^{\infty} \alpha_{n i}\left\|J T_{i}^{n} x_{n}\right\|^{2} \\
& -\alpha_{n k} \alpha_{n j} g\left(\left\|J T_{k}^{n} x_{n}-J T_{j}^{n} x_{n}\right\|\right)+2 \rho f\left(x^{*}\right) \\
= & \sum_{i=0}^{\infty} \alpha_{n i} \phi\left(x^{*}, T_{i}^{n} x_{n}\right)+2 \rho f\left(x^{*}\right)-\alpha_{n k} \alpha_{n j} g\left(\left\|J T_{k}^{n} x_{n}-J T_{j}^{n} x_{n}\right\|\right) \\
\leq & \sum_{i=0}^{\infty} \alpha_{n i} k_{n i} \phi\left(x^{*}, x_{n}\right)+2 \rho f\left(x^{*}\right)-\alpha_{n k} \alpha_{n j} g\left(\left\|J T_{k}^{n} x_{n}-J T_{j}^{n} x_{n}\right\|\right) \\
\leq & G\left(x^{*}, J x_{n}\right)+\left(k_{n}-1\right) M_{n}-\alpha_{n k} \alpha_{n j} g\left(\left\|J T_{k}^{n} x_{n}-J T_{j}^{n} x_{n}\right\|\right) .
\end{aligned}
$$


Taking $k=0$ and for any $j$ in (3.10), we have

$$
\alpha_{n 0} \alpha_{n j} g\left(\left\|J x_{n}-J T_{j}^{n} x_{n}\right\|\right) \leq G\left(x^{*}, J x_{n}\right)-G\left(x^{*}, J u_{n}\right)+\left(k_{n}-1\right) M_{n} \rightarrow 0 .
$$

It follows from the property of $g$ that

$$
\lim _{n \rightarrow \infty}\left\|J x_{n}-J T_{j}^{n} x_{n}\right\|=0
$$

Step 4. Now we prove that $p \in \Omega$.

(a) First, we prove that $p \in \bigcap_{i=0}^{\infty} F\left(T_{i}\right)$.

Since $x_{n} \rightarrow p$ and $J$ is uniformly norm-to-norm continuous on bounded sets, we see that

$$
\lim _{n \rightarrow \infty}\left\|J x_{n}-J p\right\|=0
$$

We observe from (3.11) and (3.12) that

$$
\left\|J T_{j}^{n} x_{n}-J p\right\| \leq\left\|J x_{n}-J T_{j}^{n} x_{n}\right\|+\left\|J x_{n}-J p\right\| \rightarrow 0, \quad n \rightarrow \infty .
$$

Since $J^{-1}$ is hemi-continuous, it follows that $T_{j}^{n} x_{n} \rightarrow p$. On the other hand, since

$$
\left|\left\|T_{j}^{n} x_{n}\right\|-\|p\|\right|=\left|\left\|J T_{j}^{n} x_{n}\right\|-\|J p\|\right| \leq\left\|J T_{j}^{n} x_{n}-J p\right\|,
$$

and this implies that $\left\|T_{j}^{n} x_{n}\right\| \rightarrow\|p\|$ as $n \rightarrow \infty$. Since $E$ enjoys the Kadec-Klee property, we obtain that

$$
\lim _{n \rightarrow \infty}\left\|T_{j}^{n} x_{n}-p\right\|=0
$$

Note that

$$
\left\|T_{j}^{n+1} x_{n}-p\right\| \leq\left\|T_{j}^{n+1} x_{n}-T_{j}^{n} x_{n}\right\|+\left\|T_{j}^{n} x_{n}-p\right\| .
$$

It follows from the asymptotic regularity of $T$ and (3.13) that

$$
\lim _{n \rightarrow \infty}\left\|T_{j}^{n+1} x_{n}-p\right\|=0 .
$$

That is, $T_{j} T_{j}^{n} x_{n}-p \rightarrow 0$ as $n \rightarrow \infty$. It follows from the closeness of $T_{j}$ that $T_{j} p=p, \forall j \in \mathbb{N}$, i.e., $p \in \bigcap_{i=0}^{\infty} F\left(T_{i}\right)$.

(b) Next, we prove that $p \in \bigcap_{k=1}^{m} \mathrm{EP}\left(F_{k}\right)$.

From (3.2), we obtain

$$
\begin{aligned}
\phi\left(x^{*}, u_{n}\right) & =\phi\left(x^{*}, \theta_{n}^{m} y_{n}\right)=\phi\left(x^{*}, T_{r_{m}, n}^{F_{m}} \theta_{n}^{m-1} y_{n}\right) \\
& \leq \phi\left(x^{*}, \theta_{n}^{m-1} y_{n}\right) \leq \cdots \leq \phi\left(x^{*}, y_{n}\right) \\
& \leq \phi\left(x^{*}, x_{n}\right)+\left(k_{n}-1\right) M_{n} .
\end{aligned}
$$

Next, we show that $\theta_{n}^{k} y_{n} \rightarrow p$ as $n \rightarrow \infty$, for each $k \in\{0,1, \ldots, m\}$. 
We have proved that $k=m, \theta_{n}^{k} y_{n}=u_{n} \rightarrow p$.

Suppose that $\theta_{n}^{k} y_{n} \rightarrow p$ as $n \rightarrow \infty$ for some $k$. Since $x^{*} \in \bigcap_{k=1}^{m} \operatorname{EP}\left(F_{k}\right)=\bigcap_{k=1}^{m} F\left(T_{r_{k, n}}^{F_{k}}\right)$ for all $n \geq 1$, it follows from Lemma 2.14 that

$$
\begin{aligned}
\phi\left(\theta_{n}^{k} y_{n}, \theta_{n}^{k-1} y_{n}\right) & =\phi\left(T_{r_{k, n}}^{F_{k}} \theta_{n}^{k-1} y_{n}, \theta_{n}^{k-1} y_{n}\right) \\
& \leq \phi\left(x^{*}, \theta_{n}^{k-1} y_{n}\right)-\phi\left(x^{*}, \theta_{n}^{k} y_{n}\right) \\
& \leq \phi\left(x^{*}, x_{n}\right)-\phi\left(x^{*}, \theta_{n}^{k} y_{n}\right)+\left(k_{n}-1\right) M_{n} .
\end{aligned}
$$

Hence, we have

$$
\lim _{n \rightarrow \infty} \phi\left(\theta_{n}^{k} y_{n}, \theta_{n}^{k-1} y_{n}\right)=0
$$

From (2.5), we see that $\left\|\theta_{n}^{k} y_{n}\right\|-\left\|\theta_{n}^{k-1} y_{n}\right\| \rightarrow 0$ as $n \rightarrow \infty$. From assumption, we have $\theta_{n}^{k} y_{n} \rightarrow p$ as $n \rightarrow \infty$, so

$$
\left\|\theta_{n}^{k-1} y_{n}\right\| \rightarrow\|p\| \quad \text { as } n \rightarrow \infty
$$

It follows that

$$
\left\|J \theta_{n}^{k-1} y_{n}\right\| \rightarrow\|J p\| \quad \text { as } n \rightarrow \infty .
$$

This implies that $\left\{\left\|J \theta_{n}^{k-1} y_{n}\right\|\right\}_{n=0}^{\infty}$ is bounded in $E^{*}$. Since $E$ is reflexive, and so $E^{*}$ is reflexive, we can then assume that $J \theta_{n}^{k-1} y_{n} \rightarrow f_{k-1} \in E^{*}$. In view of reflexivity of $E$, we see that $J(E)=$ $E^{*}$. Hence, there exists $x^{k-1} \in E$ such that $J x^{k-1}=f_{k-1}$. Since

$$
\begin{aligned}
\phi\left(\theta_{n}^{k} y_{n}, \theta_{n}^{k-1} y_{n}\right) & =\left\|\theta_{n}^{k} y_{n}\right\|^{2}-2\left\langle\theta_{n}^{k} y_{n}, J \theta_{n}^{k-1} y_{n}\right\rangle+\left\|\theta_{n}^{k-1} y_{n}\right\|^{2} \\
& =\left\|\theta_{n}^{k} y_{n}\right\|^{2}-2\left\langle\theta_{n}^{k} y_{n}, J \theta_{n}^{k-1} y_{n}\right\rangle+\left\|J \theta_{n}^{k-1} y_{n}\right\|^{2} .
\end{aligned}
$$

Taking $\liminf _{n \rightarrow \infty}$ for both sides of the equality above, yields that

$$
\begin{aligned}
0 & \geq\|p\|^{2}-2\left\langle p, f_{k-1}\right\rangle+\left\|f_{k-1}\right\|^{2} \\
& =\|p\|^{2}-2\left\langle p, J x^{k-1}\right\rangle+\left\|J x^{k-1}\right\|^{2} \\
& =\|p\|^{2}-2\left\langle p, J x^{k-1}\right\rangle+\left\|x^{k-1}\right\|^{2} \\
& =\phi\left(p, x^{k-1}\right) .
\end{aligned}
$$

That is, $p=x^{k-1}$. This implies that $f_{k-1}=J p$ and so $J \theta_{n}^{k-1} y_{n} \rightarrow J p$. It follows from $\lim _{n \rightarrow \infty}\left\|J \theta_{n}^{k-1} y_{n}\right\|=\|J p\|$ and Kadec-Klee property of $E^{*}$ (this is because $E^{*}$ is uniformly convex) that

$$
J \theta_{n}^{k-1} y_{n} \rightarrow J p
$$

Note that $J^{-1}: E^{*} \rightarrow E$ is hemi-continuous (this is because $E$ is a uniformly smooth and strictly convex Banach space with a strictly convex dual $E^{*}$ ), it follows that $\theta_{n}^{k-1} y_{n} \rightarrow p$. 
Since (3.14) and $E$ have the Kadec-Klee property, we obtain that

$$
\lim _{n \rightarrow \infty} \theta_{n}^{k-1} y_{n}=p
$$

Hence, $\lim _{n \rightarrow \infty} \theta_{n}^{k} y_{n}=p$ and $\lim _{n \rightarrow \infty} J \theta_{n}^{k} y_{n}=J p$, for each $k \in\{0,1, \ldots, m\}$. That is,

$$
\lim _{n \rightarrow \infty}\left\|\theta_{n}^{k} y_{n}-\theta_{n}^{k-1} y_{n}\right\|=0, \quad k=1,2, \ldots, m
$$

and

$$
\lim _{n \rightarrow \infty}\left\|J \theta_{n}^{k} y_{n}-J \theta_{n}^{k-1} y_{n}\right\|=0, \quad k=1,2, \ldots, m .
$$

Since $\liminf _{n \rightarrow \infty} r_{k, n}>0, k=1,2, \ldots, m$,

$$
\lim _{n \rightarrow \infty} \frac{\left\|J \theta_{n}^{k} y_{n}-J \theta_{n}^{k-1} y_{n}\right\|}{r_{k, n}}=0
$$

By Lemma 2.13, we have that for each $k=1,2, \ldots, m$,

$$
F_{k}\left(\theta_{n}^{k} y_{n}, y\right)+\frac{1}{r_{k, n}}\left\langle y-\theta_{n}^{k} y_{n}, J \theta_{n}^{k} y_{n}-J \theta_{n}^{k-1} y_{n}\right\rangle \geq 0, \quad \forall y \in C .
$$

Furthermore, using (A2), we obtain

$$
\frac{1}{r_{k, n}}\left\langle y-\theta_{n}^{k} y_{n}, J \theta_{n}^{k} y_{n}-J \theta_{n}^{k-1} y_{n}\right\rangle \geq F_{k}\left(y, \theta_{n}^{k} y_{n}\right)
$$

By (A4), (3.15) and $\theta_{n}^{k} y_{n} \rightarrow p$, we have for each $k=1,2, \ldots, m$,

$$
F_{k}(y, p) \leq 0, \quad \forall y \in C
$$

For fixed $y \in C$, let $z_{t}=t y+(1-t) p$ for all $t \in(0,1]$. This implies that $z_{t} \in C$. This yields that $F_{k}\left(z_{t}, p\right) \leq 0$. It follows from (A1) and (A4) that

$$
0=F_{k}\left(z_{t}, z_{t}\right) \leq t F_{k}\left(z_{t}, y\right)+(1-t) F_{k}\left(z_{t}, p\right) \leq t F_{k}\left(z_{t}, y\right)
$$

and hence

$$
0 \leq F_{k}\left(z_{t}, y\right)
$$

From condition (A3), we obtain

$$
F_{k}(p, y) \geq 0, \quad \forall y \in C
$$

This implies that $p \in \operatorname{EP}\left(F_{k}\right), k=1,2, \ldots, m$. Thus, $p \in \bigcap_{k=1}^{m} \operatorname{EP}\left(F_{m}\right)$.

Hence, we have $p \in \Omega=\left(\bigcap_{k=1}^{m} \operatorname{EP}\left(F_{m}\right)\right) \cap\left(\bigcap_{i=0}^{\infty} F\left(T_{i}\right)\right)$.

Step 5. Finally, we prove that $p=\Pi_{\Omega}^{f} x_{0}$. 
Since $\Omega=\left(\bigcap_{k=1}^{m} \operatorname{EP}\left(F_{m}\right)\right) \cap\left(\bigcap_{i=0}^{\infty} F\left(T_{i}\right)\right)$ is a closed and convex set, from Lemma 2.6, we know that $\Pi_{\Omega}^{f} x_{0}$ is single-valued and denoted $\omega=\Pi_{\Omega}^{f} x_{0}$. Since $x_{n}=\Pi_{C_{n}}^{f} x_{0}$ and $\omega \in \Omega \subset$ $C_{n}$, we have

$$
G\left(x_{n}, J x_{0}\right) \leq G\left(\omega, J x_{0}\right), \quad \forall n \geq 0 .
$$

We know that $G(\xi, \phi)$ is convex and lower semi-continuous with respect to $\xi$ when $\phi$ is fixed. This implies that

$$
G\left(p, J x_{0}\right) \leq \liminf _{n \rightarrow \infty} G\left(x_{n}, J x_{0}\right) \leq \limsup _{n \rightarrow \infty} G\left(x_{n}, J x_{0}\right) \leq G\left(\omega, J x_{0}\right)
$$

From the definition of $\Pi_{\Omega}^{f} x_{0}$ and $p \in \Omega$, we see that $p=\omega$. This completes the proof.

Corollary 3.2 Let E be a uniformly smooth and strictly convex Banach space, which has the Kadec-Klee property, and let $C$ be a nonempty closed convex subset of E. For each $k=$ $1,2, \ldots, m$, let $F_{k}$ be a bifunction from $C \times C$ satisfying (A1)-(A4), and let $\left\{T_{i}\right\}_{i=0}^{\infty}: C \rightarrow C$, $\forall i \in \mathbb{N}$ be an infinite family of closed and asymptotically quasi- $\phi$-nonexpansive mappings with sequence $\left\{k_{n i}\right\} \subset[1, \infty), k_{n i} \rightarrow 1$ as $n \rightarrow \infty$, where $T_{0}=I$. Assume that $T_{i}, \forall i \in \mathbb{N}$ is asymptotically regular on $C$, and $\Omega=\left(\bigcap_{i=0}^{\infty} F\left(T_{i}\right)\right) \cap\left(\bigcap_{k=1}^{m} \operatorname{EP}\left(F_{k}\right)\right)$ is nonempty and bounded. Suppose that $\left\{x_{n}\right\}_{n=0}^{\infty}$ is generated by $x_{0} \in C, C_{1}=C, x_{1}=\Pi_{C_{1}}^{f} x_{0}$,

$$
\left\{\begin{array}{l}
y_{n}=J^{-1}\left\{\sum_{i=0}^{\infty} \alpha_{n i} J T_{i}^{n} x\right\} \\
u_{n}=T_{r_{m, n}}^{F_{m}} T_{r_{m-1, n}}^{F_{m-1}} \cdots T_{r_{2, n}}^{F_{2}} T_{r_{1, n}}^{F_{1}} y_{n}, \\
C_{n+1}=\left\{z \in C_{n}: \phi\left(z, J u_{n}\right) \leq \phi\left(z, J x_{n}\right)+\left(k_{n}-1\right) M_{n}\right\} \\
x_{n+1}=\Pi_{C_{n+1}} x_{0},
\end{array}\right.
$$

where $J$ is the duality mapping on $E, M_{n}=\sup \left\{\phi\left(z, x_{n}\right): z \in \Omega\right\}$ for each $n \geq 1, k_{n}=$ $\sup _{i \geq 0}\left\{k_{n i}\right\},\left\{\alpha_{n i}\right\}$ is a real sequence in $[0,1]$ and $\left\{r_{k, n}\right\}_{n=1}^{\infty} \subset(0, \infty), k=1,2, \ldots, m$, satisfying the following conditions:
(a) $\sum_{i=0}^{\infty} \alpha_{n i}=1, \quad \forall n \geq 1$;
(b) $\quad \liminf _{n \rightarrow \infty} \alpha_{n 0} \alpha_{n i}>0, \quad \forall i \in \mathbb{N}$;
(c) $\quad \liminf _{n \rightarrow \infty} r_{k, n}>0$.

Then the sequence $\left\{x_{n}\right\}$ converges strongly to $\Pi_{\Omega} x_{0}$.

\section{Competing interests \\ The authors declare that they have no competing interests.}

\section{Authors' contributions}

All authors read and approved the final manuscript.

\section{Acknowledgements}

The authors would like to express their sincere thanks to the anonymous referee and the editor for their valuable suggestions and comments, which greatly improved the original version of the manuscript.

Received: 28 December 2012 Accepted: 1 August 2013 Published: 20 August 2013 


\section{References}

1. Yang, L, Zhao, FH, Kim, JK: Hybrid projection method for generalized mixed equilibrium problem and fixed point problem of infinite family of asymptotically quasi- $\phi$-nonexpansive mappings in Banach spaces. Appl. Math. Comput. $218,6072-6082(2012)$

2. Shehu, Y: Hybrid iterative scheme for fixed point problem, infinite systems of equilibrium and variational inequality problems. Comput. Math. Appl. 63, 1089-1103 (2012)

3. Takahashi, W: Nonlinear Functional Analysis. Yokohama Publishers, Yokohama (2000)

4. Cioranescu, I: Geometry of Banach Spaces, Duality Mappings and Nonlinear Problems. Kluwer Academic, Dordrecht (1990)

5. Reich, S: A weak convergence theorem for the alternating method with Bregman distance. In: Kartsatos, AG (ed.) Theory and Applications of Nonlinear Operator of Accretive and Monotone Type. Dekker, New York (1996)

6. Butnariu, D, Reich, S, Zaslavski, AJ: Asymptotic behavior of relatively nonexpansive operators in Banach spaces. J. Appl. Anal. 7, 151-174 (2001)

7. Butnariu, D, Reich, S, Zaslavski, AJ: Weak convergence of orbits of nonlinear operators in reflexive Banach spaces. Numer. Funct. Anal. Optim. 24, 489-508 (2003)

8. Censor, Y, Reich, S: Iterations of paracontractions and firmly nonexpansive operators with applications to feasibility and optimization. Optimization 37, 323-339 (1996)

9. Qin, XL, Cho, YJ, Kang, SM: Convergence theorems of common elements for equilibrium problems and fixed point problems in Banach spaces. J. Comput. Appl. Math. 225, 20-30 (2009)

10. Qin, $\mathrm{XL}$, Cho, YJ, Kang, SM, Zhou, H: Convergence of a modified Halpern-type iteration algorithm for quasi- $\phi$-nonexpansive mappings. Appl. Math. Lett. 22, 1051-1055 (2009)

11. Zhou, HY, Gao, GL, Tan, B: Convergence theorems of a modified hybrid algorithm for a family of quasi- $\boldsymbol{\phi}$-asymptotically nonexpansive mappings. J. Appl. Math. Comput. 32, 453-464 (2010)

12. Chen, YQ, Kim, JK: Existence results for systems of vector equilibrium problems. J. Glob. Optim. 35(1), 71-83 (2006)

13. Alber, Yl: Metric and generalized projection operators in Banach spaces: properties and applications. In: Kartsatos, AG (ed.) Theory and Applications of Nonlinear Operator of Accretive and Monotone Type, pp. 15-50. Dekker, New York (1996)

14. Alber, YI, Reich, S: An iterative method for solving a class of nonlinear operator equations in Banach spaces. Panam. Math. J. 4, 39-54 (1994)

15. Wu, KQ, Huang, NJ: The generalized f-projection operator with application. Bull. Aust. Math. Soc. 73, 307-317 (2006)

16. Fan, JH, Liu, X, Li, JL: Iterative schemes for approximating solutions of generalized variational inequalities in Banach space. Nonlinear Anal. 70, 3997-4007 (2009)

17. Li, X, Huang, N, O'Regan, D: Strong convergence theorems for relatively nonexpansive mappings in Banach spaces with applications. Comput. Math. Appl. 60, 1322-1331 (2010)

18. Deimling, K: Nonlinear Function Analysis. Springer, Berlin (1985)

19. Kim, JK: Strong convergence theorems by hybrid projection methods for equilibrium problems and fixed point problems of the asymptotically quasi- $\phi$-nonexpansive mappings. Fixed Point Theory Appl. (2011). doi:10.1186/1687-1812-2011-10

20. Blum, E, Oettli, W: From optimization and variational inequalities to equilibrium problems. Math. Stud. 63, 123-145 (1994)

21. Takahashi, W, Zembayashi, K: Strong and weak convergence theorems for equilibrium problems and relatively nonexpansive mappings in Banach spaces. Nonlinear Anal. 70, 45-47 (2009)

doi:10.1186/1687-1812-2013-221

Cite this article as: Wang et al.: Strong convergence theorems for fixed point problems of infinite family of asymptotically quasi- $\phi$-nonexpansive mappings and a system of equilibrium problems. Fixed Point Theory and Applications 2013 2013:221. 